\title{
A dual-plane approach for surgical treatment of pseudoaneurysm with arteriovenous fistula in hemodialysis patients
}

\author{
Ji Min Kim ${ }^{1}$, Min Sung Tak ${ }^{1}$, Jin Seok Kang ${ }^{1}$, Chul Moon ${ }^{2}$ \\ Departments of ${ }^{1}$ Plastic and Reconstructive Surgery and ${ }^{2}$ General Surgery, Soonchunhyang University Seoul Hospital, Seoul, Korea
}

\begin{abstract}
Background We report the efficacy of a dual-plane approach using a Dufourmentel skin flap with a purse-string suture of the de-epithelized dermis to manage pseudoaneurysm at the vascular access site for hemodialysis.

Methods A retrospective analysis was conducted of 61 patients from 2013 to 2018 with pseudoaneurysms at the arteriovenous fistula or graft who were treated with rhomboid excision, vessel repair with a purse-string suture, and a full-thickness Dufourmentel skin flap. The success rate was defined as the probability of complete wound closure and intact vascular access patency without infection or other complications.

Results The success rate was $93.4 \%$ at 6 months postoperatively. Complications included newly occurring pseudoaneurysms $(n=2)$, wound dehiscence $(n=1)$ and bleeding $(n=1)$. There were no complications such as stenosis or thrombosis from the procedure.

Conclusions A dual-plane approach using a Dufourmentel skin flap with a purse-string suture for vessel repair was shown to be a favorable option for managing stable, small (diameter $<2 \mathrm{~cm}$ ) pseudoaneurysms without infection, rapid expansion, or patency issues of the vascular access.
\end{abstract}

\section{Keywords Pseudoaneurysm / Hemodialysis / Surgical flap}

Received: October 27, 2020 • Revised: February 5, $2021 \bullet$ Accepted: February 24, 2021

pISSN: 2234-6163 • elSSN: 2234-6171 • https://doi.org/10.5999/aps.2020.02068• Arch Plast Surg 2021;48:287-292
Correspondence: Min Sung Tak Department of Plastic and Reconstructive Surgery, Soonchunhyang University Seoul Hospital, 59 Daesagwan-ro, Yongsan-gu, Seoul 04401, Korea Tel: +82-2-709-9283

Fax: +82-2-705-3687

E-mail: tarkms@naver.com

\section{INTRODUCTION}

Vascular access for hemodialysis is important enough to be considered a patient's lifeline. It is essential to ensure and maintain vascular access for hemodialysis patients to receive adequate dialysis. If the vascular access is inadequate, patients may experience a variety of negative consequences due to a lack of dialysis. Serious complications such as thrombosis, limb swelling, ischemic steal syndrome, neuropathy, infection, cardiovascular disease, and the formation of aneurysms or pseudoaneurysms that affect the patient's lifespan can occur [1]. Vascular complications such as obstruction, infection, stenosis, and aneurysms are economically and psychologically burdensome as they require hospitalization or surgery.

Pseudoaneurysm, also known as a false aneurysm, is a collection of blood that forms between the two outer layers of an artery, typically having a narrow neck and fibrous capsule, and is a complication of hemodialysis arteriovenous graft (AVG) or arteriovenous fistula (AVF) associated with an increased risk of thrombosis, bleeding, infection, pain, and hemodialysis failure 
[2]. A pseudoaneurysm usually occurs from repeated trauma that causes blood to leak into surrounding tissues. As the hematoma resolves, scars and fibrous capsules form externally. Improper use of an AVF, such as frequent cannulation and external mechanical pressure at the same site, can lead to aneurysms [1]. Surgical interventions for pseudoaneurysms include ligation, end-to-end AVF, excision and repair, endovascular procedures, and embolization. However, no "gold standard" exists for the management of pseudoaneurysms [2-6]. We present our experiences with the surgical management of pseudoaneurysms and report the efficacy of a dual-plane approach using a Dufourmentel skin flap with a purse-string suture for vessel wall reinforcement.

\section{METHODS}

\section{Study design}

The study design was a single-center, retrospective chart review, focusing on the efficacy and complications of the dual-plane approach for pseudoaneurysms. The study was approved by Institutional Review Board of Soonchunhyang University Hospital (IRB No. 2020-11-008-001). Written informed consent was obtained from all patients.

\section{Inclusion and exclusion criteria}

We included patients who underwent treatment at our institution from December 2013 to December 2018 with the dualplane approach for the treatment of pseudoaneurysms at the hemodialysis access site. The exclusion criteria were patients with (1) multiple pseudoaneurysms; (2) an infected pseudoaneurysm, or (3) a pseudoaneurysm larger than $2 \mathrm{~cm}$.

A retrospective analysis was performed of cases of pseudoaneurysm treated with a dual-plane approach and data were obtained from the vascular database and medical records. Data included patient demographics, clinical characteristics, postopera- tive complications within 6 months, and the follow-up period.

Pseudoaneurysm was clinically diagnosed before surgery according to the location and site of abnormally localized vascular dilation. Percutaneous transluminal angioplasty for the treatment of eight patients with downstream stenosis was performed at least 2 weeks prior to surgery. Hemodialysis was performed by puncturing another site of the access during the healing period of the flap. Postoperative ultrasonography was performed 1 month postoperatively to evaluate the vascular access site before the start of hemodialysis. Surgical success was defined as wound healing and intact vascular access patency without complications.

\section{Surgical technique}

All patients received local anesthesia using $2 \%$ lidocaine mixed with 1:100,000 epinephrine. A $1.5-\mathrm{cm}$ incision on proximal portion of the vascular access was properly made as far away from the needling site as possible and a vascular clamp was placed. The preoperative design of the Dufourmentel flap was essential for a successful outcome. First, we designed a rhombus at the site of the pseudoaneurysm for excision. The long diagonal axis of the rhombus was designed parallel to the vein/graft direction and we designed a larger rhomboid than the pseudoaneurysm area. Next, we designed a Dufourmentel flap from the adjacent normal skin, which did not interfere with hemodialysis access, to cover the excision site (Fig. 1). Full-thickness skin, including sufficient subcutaneous tissue, was raised and rotated to cover the defect without tension. To prevent excessive bleeding, a vascular clamp was applied via separate incisions proximal to the pseudoaneurysm. De-epithelization was performed carefully to avoid damaging the underlying pseudoaneurysm vessel wall (Fig. 2). We found that the top area of the pseudoaneurysm capsule was thin, unhealthy, and pliable, but the bottom areas of the pseudoaneurysm capsule were relatively thick and healthy (Fig. 3). Thus, a purse-string suture using a nonabsorbable
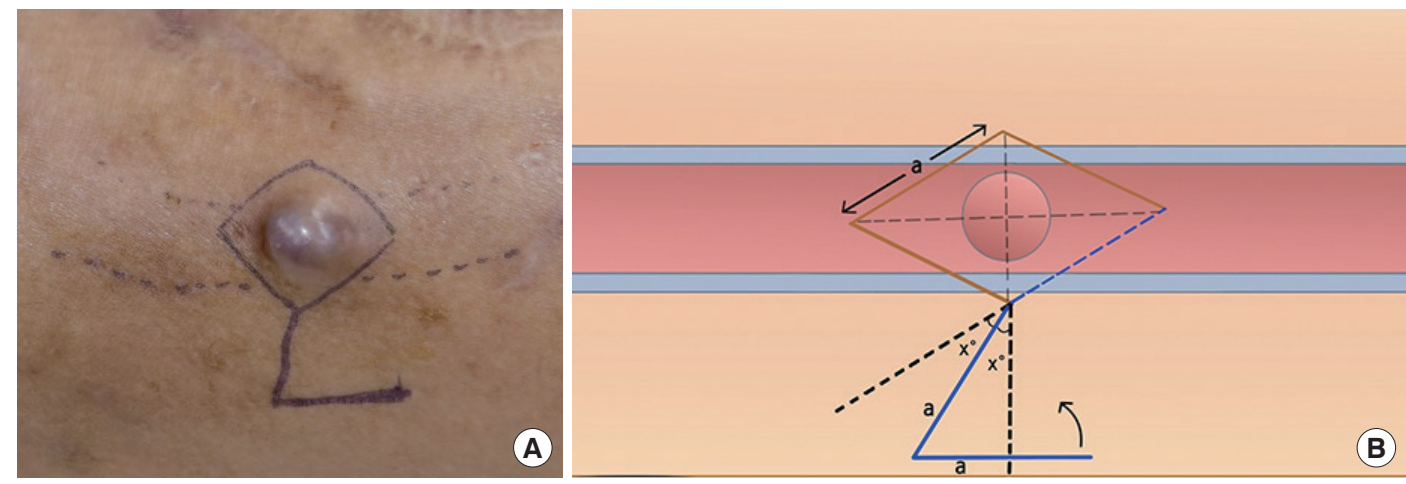

Fig. 1. Preoperative design of a Dufourmentel skin flap. A pseudoaneurysm with a skin-thinning lesion is shown on an arteriovenous fistula. (A) Preoperative photograph. (B) Schematic of the Dufourmentel skin flap. 
monofilament suture (5-0 Prolene) was performed on a healthy pseudoaneurysm capsule with de-epithelized dermis covering the capsule, and the unhealthy lesion was trimmed (Fig. 4). Through this procedure, we could reduce the size of the pseudoaneurysm and properly reinforce the pseudoaneurysm wall. Finally, we rotated the Dufourmentel flap to cover the excised area and sutured with monofilament interrupted sutures. Primary closure of the donor site was done, and the vascular clamp was released (Fig. 5). Because the skin problems were treated through a Dufourmentel skin flap, and vessel problems were treated through a purse-string suture of the pseudoaneurysm vessel wall, we termed this technique the "dual-plane approach."

\section{RESULTS}

A total of 71 patients who underwent the dual-plane approach

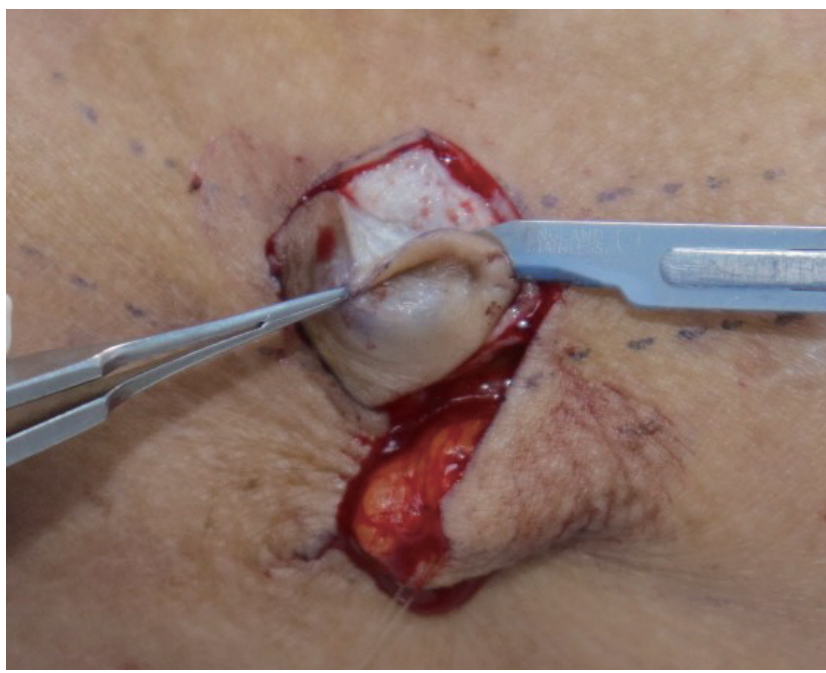

Fig. 2. De-epithelization was performed in the rhombus area, taking care not to damage the underlying pseudoaneurysm capsule.

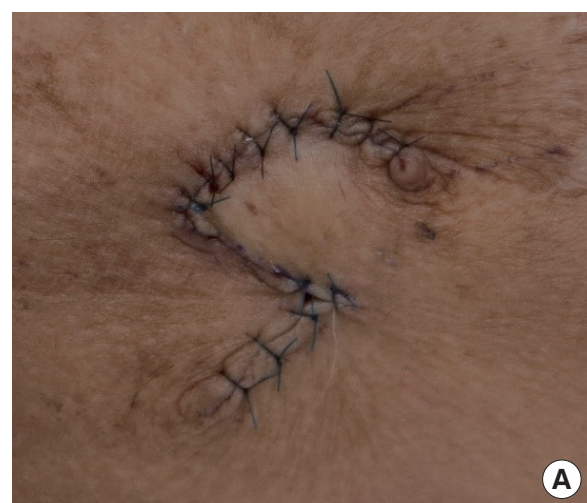

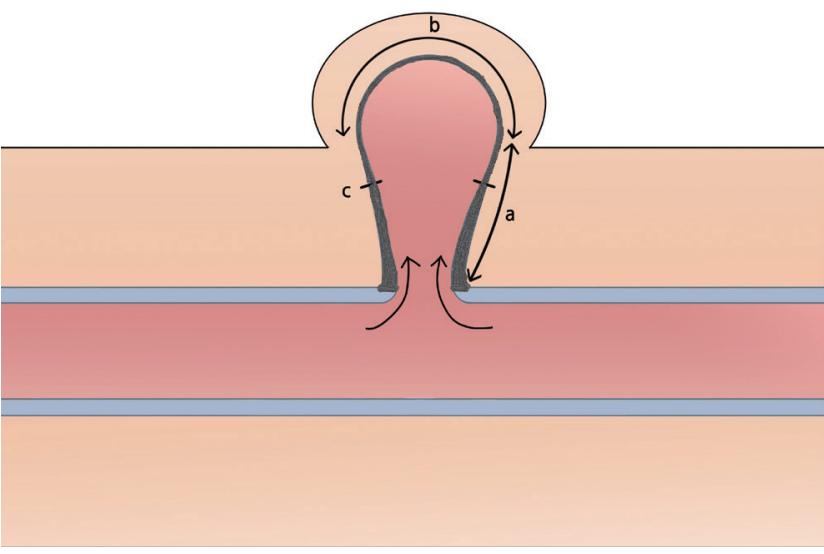

Fig. 3. Schematic finding of a pseudoaneurysm. The bottom of the pseudoaneurysm capsule (a) was relatively thick and strong enough to endure suture tension, whereas the central area of the pseudoaneurysm capsule (b) was pliable, thin, and nonhealthy. Purse-string suturing was performed on the healthy pseudoaneurysm capsule (c).

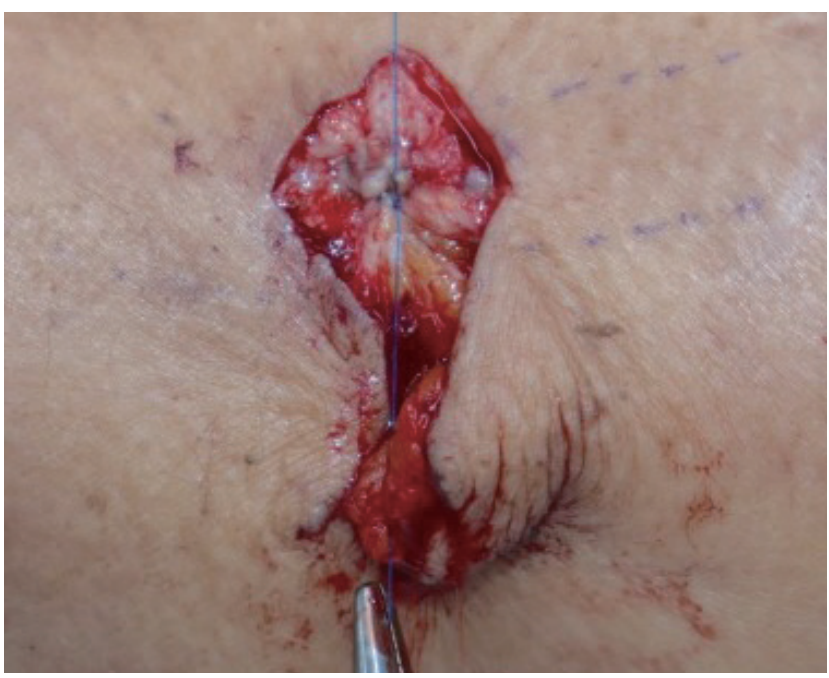

Fig. 4. Purse-string suturing was performed in the healthy pseudoaneurysm capsule with de-epithelized dermis that covered the capsule using No. 5-0 Prolene, and the nonhealthy lesion was trimmed.

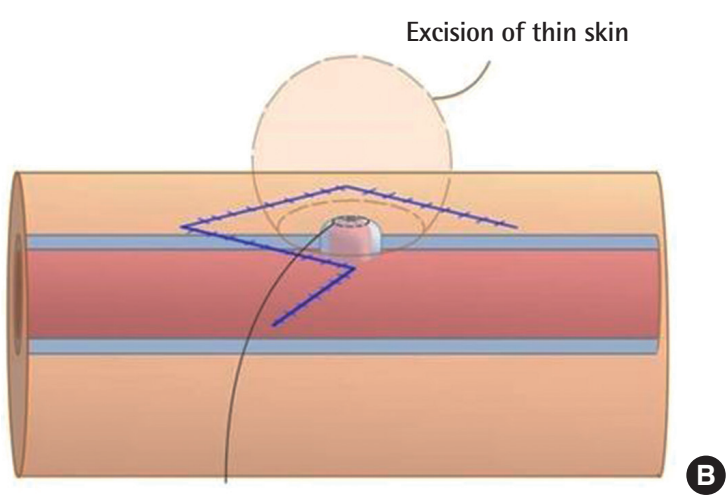

Purse-string suture of de-epithelized dermis

Fig. 5. Postoperative findings. The area where the pseudoaneurysm was present was double-covered with a purse-string suture and the Dufourmentel flap. (A) Postoperative photograph. (B) Schematic of the reinforced pseudoaneurysm wall. 
Table 1. Operative data of the pseudoaneurysms

\begin{tabular}{lc}
\hline Variable & Mean (range) \\
\hline Operation time (min) & $49.40(34-70)$ \\
Diameter of pseudoaneurysm (cm) & $1.37(1-2)$ \\
Follow-up period (mon) & $13.10(6-30)$ \\
\hline
\end{tabular}

for the treatment of a pseudoaneurysm at the hemodialysis access site were identified. Ten patients were excluded, and the 61 patients included in this study comprised 21 men and 40 women (mean age, 64 years). The median duration from the initiation of hemodialysis to surgery was 68.4 months (range, 3-324 months).

The average operation time was 49.4 minutes and the mean pseudoaneurysm diameter was $1.37 \mathrm{~cm}$. The median follow-up period was 13.1 months (Table 1).

Complications that occurred within 6 months postoperatively included pseudoaneurysm recurrence $(n=2)$, wound dehiscence $(\mathrm{n}=1)$, and bleeding $(\mathrm{n}=1)$, as shown in Table 2. Cases of pseudoaneurysm recurrence were treated by reoperation with the same method. Wound dehiscence and bleeding were controlled by conservative treatment without secondary procedures. All complications occurred in cases with pseudoaneurysm as well as another preoperative lesion, such as skin erosion or ulceration $(n=4)$. The success rate at 6 months postoperatively was $93.4 \%$.

\section{DISCUSSION}

In Korea, the number of hemodialysis patients, especially elderly patients with diabetes, is increasing rapidly. At the end of 2014 , the number of patients with end-stage renal disease in Korea was 86,674 , of whom 57,256 were hemodialysis patients [7]. As the number of dialysis patients increases, so do the societal costs. Approximately $16 \%$ to $25 \%$ of total annual hospitalizations for hemodialysis patients are associated with complications of vascular access [8].

Pseudoaneurysm is a complication that occurs in $2 \%$ to $10 \%$ of AVGs and in a smaller proportion of AVFs [9]. Pseudoaneurysms of prosthetic grafts mainly arise from repeated cannulation in the same area, and blood leakage occurs, forming a kind of capsule under the support of surrounding tissues. Skin tension increases due to the expansion of the underlying pseudoaneurysm as the skin and subcutaneous tissue are damaged by repeated cannulation. This causes the skin to become thinner and eroded, resulting in more necrosis and exposure of the graft or fistula, which is at greater risk of rupture and fatal bleeding. In our study, all pseudoaneurysm patients had thinned skin at the lesion. Similar studies published by Georgiadis et al. [10] and Al-Thani et al. [1] also reported thinning and erosion of the skin
Table 2. Postoperative complications of hemodialysis patients with pseudoaneurysms

\begin{tabular}{lcc}
\hline Variable & AVF & AVG \\
\hline Complications (within 6 months) & & 1 \\
Wound dehiscence & & \\
Infection & & 1 \\
Bleeding & 1 & 1 \\
Pseudoaneurysm recurrence & 1 & \\
\hline
\end{tabular}

AVF, arteriovenous fistula; AVG, arteriovenous graft.

in most cases of aneurysmal AVFs.

The management of pseudoaneurysm, which complicates AVFs and AVGs, can be divided into surgical approaches and endovascular interventions. Endovascular interventions through ultrasound-guided stent grafts, embolization, or thrombin injection are minimally invasive alternatives, but can only be used in stable pseudoaneurysms. Shah et al. [9] reported that the endovascular stent graft repair of pseudoaneurysms allows immediate use of the vascular access site as well as the identification and treatment of associated stenosis. However, they also reported that the principal limitation of stent graft repair for pseudoaneurysm with skin erosion was a high rate of failure due to infection (25\%). They considered a bacterial colony in the pseudoaneurysm cavity at the time of treatment to be the cause of failure. Percutaneous thrombin injection has proven to be a safe and effective treatment for pseudoaneurysms. Clark and Abraham [11] successfully treated two cases of brachial artery pseudoaneurysm at the AVF site with thrombin injections. However, vascular access patency is not preserved with this method, and complications of thrombin escaping into the native circulatory system can lead to distal embolism.

Meanwhile, unstable pseudoaneurysms with infection and rapidly expanding, large skin ulcerations or perforations require surgical approaches including ligation, resection, resection, endto-end anastomosis, resection, or partial aneurysmectomy. Karabay et al. [5] performed aneurysmectomy and revascularization of the artery in 18 patients with giant aneurysms that occurred in the antecubital AVF and reported technical success in all patients. Ozcan et al. [12] demonstrated successful aneurysm resection and plication in all 16 true aneurysmal patients. Hossny [13] performed partial aneurysm resection for the salvage of autogenous AVFs with complicated venous aneurysms. This method differs from conventional methods that generally require prosthetic interposition after ligation or resection and provides the ability to conserve the benefits of autogenous access while preserving the site of future dialysis. In a systematic review of AVF aneurysmal formation, including 12 studies and 23 case reports, aneurysmectomy was the most preferred treatment option, followed by vein graft interposition, both of which were 
preferred over bypassing surgery [14].

For a relatively stable, single, small pseudoaneurysm $(<2 \mathrm{~cm}$ in diameter) and skin lesions such as erosion, necrosis, bleeding, and without infection or rapid expansion, we thought that the surgical method could be a good option. However, if aggressive methods such as excision or an interposition graft are performed first, the number of options to choose in the future will be reduced in the future when other serious vascular problems arise. In addition, it is difficult to perform endovascular interventions immediately due to skin lesions. In these cases, we hypothesized that minimally invasive surgery with a Dufourmentel skin flap and partial pseudoaneurysm resection using a pursestring suture could simultaneously resolve the pseudoaneurysm with the associated skin lesions and salvage the vascular access. Percutaneous transluminal angioplasty was generally performed at least 2 weeks prior to surgery in the presence of a venous downstream stenosis.

Jaffers and Fasola [15] reported their experiences with AVFs with ulceration/bleeding and demonstrated that minimal skin mobilization or flap advancement was successful after ulcer excision and vessel repair or a vein interposition graft. This was similar to our study in that the skin and vessel problems were solved at the same time. A pseudoaneurysm is thought to be not only a skin problem, but also injury and weakening of the vessel wall. When only the skin lesion is treated, the remaining pseudoaneurysm expands and compresses the overlying skin, causing new skin lesions such as erosion, necrosis, and recurrence. Jaffers and Fasola proposed mobilizing an aneurysm by excising an ellipse of the overlying skin along the axis of the fistula so that the proximal and distal blood vessels could be controlled. The skin suture was made directly over the pseudoaneurysm and vascular access. In this case, as the skin tension increased by flap advancement and a skin suture was placed directly above the pseudoaneurysm, there were concerns about wound healing. We used the Dufourmentel flap to rotate the healthy skin flap in the adjacent area and avoid the skin suture just above the pseudoaneurysm area to minimize the skin tension. A pursestring suture was used to reinforce the vessel wall. It is important to choose the proper location of a purse-string suture. The pseudoaneurysm walls were divided into healthy and unhealthy areas according to their location, and based on that classification, an appropriate suture location was selected. During surgery, we found that the healthy capsule wall adjacent to the graft was thicker and strong enough to endure the tension, while the unhealthy capsule wall adjacent to the skin was thinner and more pliable (Fig. 3). Therefore, the healthy vessel wall was sutured, and the unhealthy area was removed. As a result, the vessel wall could only be reconstructed in the healthy area and the pseudo- aneurysm size was reduced.

We operated on five cases of pseudoaneurysms with infected skin lesions. Three complications occurred, including recurrence, persistent infection, and wound dehiscence. In these cases, we suggest that the infection was not limited to the skin lesions, but also involved the underlying pseudoaneurysm. The infected pseudoaneurysm vessel walls were thin and not strong enough to endure the tension of the purse-string suture, resulting in a high rate of complications (60\%). Furthermore, considering the size of the skin flap, which should be larger than the pseudoaneurysm, and the extent of tension that the capsule can withstand, about a $2-\mathrm{cm}$ pseudoaneurysm is actually the maximal indication for this procedure. Therefore, we consider pseudoaneurysms with infections as a relative contraindication and these pseudoaneurysms were excluded from this study. In cases that do not satisfy the indications, we could consider an additional procedure, such as an interposition graft or end-to-end anastomosis.

In conclusion, the dual-plane approach using a Dufourmentel skin flap and purse-string suture for pseudoaneurysm vessel repair may be a reliable option for treating single small-sized pseudoaneurysms and skin lesions without infections or rapid expansion. The dual-plane approach is simple, requires a short operative time, can salvage the existing vascular access by avoiding aggressive surgical methods, and enables skin lesion excision and pseudoaneurysm vessel repair to be performed simultaneously with a high success rate.

\section{NOTES}

\section{Conflict of interest}

No potential conflict of interest relevant to this article was reported.

\section{Ethical approval}

The study was approved by the Institutional Review Board of Soonchunhyang University Hospital (IRB No. 2020-11-008001) and performed in accordance with the principles of the Declaration of Helsinki. Written informed consent was obtained.

\section{Patient consent}

The patients provided written informed consent for the publication and the use of their images.

\section{Author contribution}

Conceptualization: MS Tak, C Moon. Data curation: JM Kim, JS Kang. Project administration: JM Kim. Writing-original draft: 
JM Kim. Writing-review \& editing: JM Kim. Approval of final manuscript: all authors.

\section{ORCID}

Ji Min Kim https://orcid.org/0000-0001-5782-8958 Min Sung Tak https://orcid.org/0000-0003-4919-5171 Jin Seok Kang https://orcid.org/0000-0003-0581-0566

Chul Moon

\section{REFERENCES}

1. Al-Thani H, El-Menyar A, Al-Thani N, et al. Characteristics, management, and outcomes of surgically treated arteriovenous fistula aneurysm in patients on regular hemodialysis. Ann Vasc Surg 2017;41:46-55.

2. Ryan JM, Dumbleton SA, Doherty J, et al. Technical innovation: using a covered stent (Wallgraft) to treat pseudoaneurysms of dialysis grafts and fistulas. AJR Am J Roentgenol 2003; 180:1067-71.

3. Lo HY, Tan SG. Arteriovenous fistula aneurysm: plicate, not ligate. Ann Acad Med Singap 2007;36:851-3.

4. Sigala F, Kontis E, Sassen R, et al. Autologous surgical reconstruction for true venous hemodialysis access aneurysms: techniques and results. J Vasc Access 2014;15:370-5.

5. Karabay O, Yetkin U, Silistreli E, et al. Surgical management of giant aneurysms complicating arteriovenous fistulae. J Int Med Res 2004;32:214-7.

6. Davison BD, Polak JF. Arterial injuries: a sonographic approach. Radiol Clin North Am 2004;42:383-96.

7. Jin DC, Yun SR, Lee SW, et al. Lessons from 30 years' data of Korean end-stage renal disease registry, 1985-2015. Kidney Res Clin Pract 2015;34:132-9.

8. Lafrance JP, Rahme E, Lelorier J, et al. Vascular access-related infections: definitions, incidence rates, and risk factors. Am J Kidney Dis 2008;52:982-93.

9. Shah AS, Valdes J, Charlton-Ouw KM, et al. Endovascular treatment of hemodialysis access pseudoaneurysms. J Vasc Surg 2012;55:1058-62.

10. Georgiadis GS, Lazarides MK, Panagoutsos SA, et al. Surgical revision of complicated false and true vascular access-related aneurysms. J Vasc Surg 2008;47:1284-91.

11. Clark TW, Abraham RJ. Thrombin injection for treatment of brachial artery pseudoaneurysm at the site of a hemodialysis fistula: report of two patients. Cardiovasc Intervent Radiol 2000;23:396-400.

12. Ozcan S, Odabasi D, Kutay V. Our experiences in the surgical treatment of venous aneurysms appearing as secondary to the upper extremity arteriovenous fistula on patients with chronic renal failure. Acta Medica Mediterranea 2014;30: 569.

13. Hossny A. Partial aneurysmectomy for salvage of autogenous arteriovenous fistula with complicated venous aneurysms. J Vasc Surg 2014;59:1073-7.

14. Kordzadeh A, D’Espiney Barbara RM, Ahmad AS, et al. Donor artery aneurysm formation following the ligation of haemodialysis arteriovenous fistula: a systematic review and case reports. J Vasc Access 2015;16:5-12.

15. Jaffers GJ, Fasola CG. Experience with ulcerated, bleeding autologous dialysis fistulas. J Vasc Access 2012;13:55-60. 